\title{
Genetic predisposition to smoking is associated with risk of rheumatoid arthritis: a Mendelian randomization study
}

\author{
Yu Qian", Lingzhi Zhang ${ }^{1}$, David J. H. Wu2 ${ }^{2}$ Zhijun Xie ${ }^{1,3}$, Chengping Wen ${ }^{1,3^{*}}$ and Yingying Mao ${ }^{1,4^{*}}$ (D)
}

\begin{abstract}
Background: Although observational epidemiological studies have found that smoking is positively associated with risk of rheumatoid arthritis (RA), assessing the causality of this relationship has remained elusive because conventional observational studies are susceptible to bias such as confounding and reverse causation. Here, we applied the Mendelian randomization (MR) approach to examine the potential causal relationship between smoking and risk of RA.
\end{abstract}

Methods: Summary statistics data for RA were obtained from a meta-analysis of genome-wide association studies (GWAS), including 14,361 RA cases and 43,923 controls of European ancestry. The instrumental variables (IV) and the genetic association estimates for smoking initiation and lifetime smoking were obtained from a GWAS metaanalysis including 1,232,091 individuals and a GWAS of 462,690 individuals of European ancestry, respectively. MR analyses were performed using the inverse-variance weighted (IVW) method and supplemented with the weightedmedian method. Potential pleiotropy was assessed using the MR-Pleiotropy RESidual Sum and Outlier (MR-PRESSO) test and MR-Egger regression. Sensitivity analyses were further performed to test the robustness of the association.

Results: We found that compared with never smokers, genetic predisposition to smoking initiation was positively associated with risk of RA (odds ratio $(O R)=1.32$, 95\% confidence interval $(C l)=1.15-1.52, P=9.17 \times 10^{-5}$ using the IVW method). Similarly, genetically predicted lifetime smoking was associated with an increased risk of RA (OR $=$ $1.55,95 \% \mathrm{Cl}=1.13-2.14, P=0.007)$. Sensitivity analyses using alternative MR methods and different sets of IVs produced similar results, suggesting the robustness of our findings.

Conclusions: These results provide support for a causal association between smoking and increased risk of RA. Further studies are warranted to explain the underlying mechanisms of smoking in the development of RA.

Keywords: Mendelian randomization, Rheumatoid arthritis, Single nucleotide polymorphism, Smoking

\section{Background}

Rheumatoid arthritis (RA) is a chronic autoimmune disease that causes cartilage and bone damage, functional loss, and associated comorbidity. It affects about $1 \%$ of the population and is more prevalent in women than in

\footnotetext{
*Correspondence: wengcp@163.com; myy@zcmu.edu.cn

${ }^{1}$ School of Public Health, Zhejiang Chinese Medical University, Hangzhou 310053, Zhejiang, China

Full list of author information is available at the end of the article
}

men [1]. Although the etiology of RA remains unclear, it is thought that the interplay of genetics, environment, and the immune system plays a major role in its development [2].

Historically, observational epidemiological studies have investigated smoking as an important modifiable risk factor for RA. Though there are inconsistent findings, evidence from these observational studies generally supports a positive association of smoking with risk of RA.

\section{$\triangle B M C$}

(C) The Author(s). 2020 Open Access This article is licensed under a Creative Commons Attribution 4.0 International License, which permits use, sharing, adaptation, distribution and reproduction in any medium or format, as long as you give appropriate credit to the original author(s) and the source, provide a link to the Creative Commons licence, and indicate if changes were made. The images or other third party material in this article are included in the article's Creative Commons licence, unless indicated otherwise in a credit line to the material. If material is not included in the article's Creative Commons licence and your intended use is not permitted by statutory regulation or exceeds the permitted use, you will need to obtain permission directly from the copyright holder. To view a copy of this licence, visit http://creativecommons.org/licenses/by/4.0/. The Creative Commons Public Domain Dedication waiver (http://creativecommons.org/publicdomain/zero/1.0/) applies to the data made available in this article, unless otherwise stated in a credit line to the data. 
For example, a meta-analysis of five cohorts and 11 case-control studies involving 584,455 individuals showed a $40 \%$ higher risk of RA among ever smokers compared to never smokers [3]. Another dose-response meta-analysis of three cohorts and seven case-control studies found that compared to never smokers, the risk of RA increased by $26 \%$ for those who smoked 1 to 10 pack-years and $94 \%$ for those with more than 20 packyears [4]. However, conventional observational studies generally rely on self-reported information and are susceptible to potential confounding and reverse causation. Therefore, the causal nature of this association remains elusive.

Mendelian randomization (MR) offers a way to investigate the nature of the relationship between smoking and risk of RA. It utilizes instrumental variables (IV) such as genetic variants that act as proxies for environmental or behavioral factors to determine whether an observational association between a risk factor and an outcome is consistent with a causal effect [5]. Because genetic variants are naturally and randomly assorted during meiosis, confounding factors are anticipated to be equally distributed among different genotypes. Therefore, results from MR studies are less prone to confounding and reverse causation bias. In the present study, we applied a two-sample MR approach to examine whether genetic predisposition to smoking was associated with risk of RA.

\section{Methods}

\section{Data sources}

We performed the MR analysis with summary statistics data from published genome-wide association studies (GWAS). An overview of the study design is shown in Fig. 1. Summarized data (effect size estimates and their standard errors) for the associations between genetic variants and risk of RA was obtained from a metaanalysis of genome-wide association studies (GWAS), including 58,284 individuals of European ancestry (14,361 RA cases and 43,923 controls) [6]. Detailed information of the study has been described elsewhere [6]. Briefly, all cases were diagnosed by a board-certified rheumatologist or met the 1987 criteria of the American College of Rheumatology for diagnosis of RA [7].

\section{Selection of instrumental variables}

The genetic IVs related to smoking initiation (ever being a regular smoker vs. never being a regular smoker) were obtained from a GWAS meta-analysis, including 1,232, 091 individuals of European ancestry [8]. This study identified 378 conditionally independent single nucleotide polymorphisms (SNPs) associated with smoking initiation at genome-wide significance threshold $(P<5 \times$ $10^{-8}$ ). All 378 SNPs together accounted for $2.3 \%$ of the variance in smoking initiation. However, 34 SNPs were not available in the summary statistics data for RA. A proxy variant in linkage disequilibrium $\left(r^{2}>0.9\right)$ with the specified genetic variant was identified for 23 of the missing SNPs. Therefore, a total of 367 SNPs were used as IVs for smoking initiation in the present study.

As an additional analysis, we used 126 independent SNPs associated with lifetime smoking at genome-wide significance from a GWAS of 462,690 individuals of European ancestry [9]. The lifetime smoking index captured individual aspects of smoking status, smoking duration, heaviness, and cessation among ever smokers. These SNPs were broadly distinct from the SNPs associated with smoking initiation, and strongly related to the control of lung cancer [9]. Among them, 124 (including 5 proxy variants) were available in the summary statistics for RA.

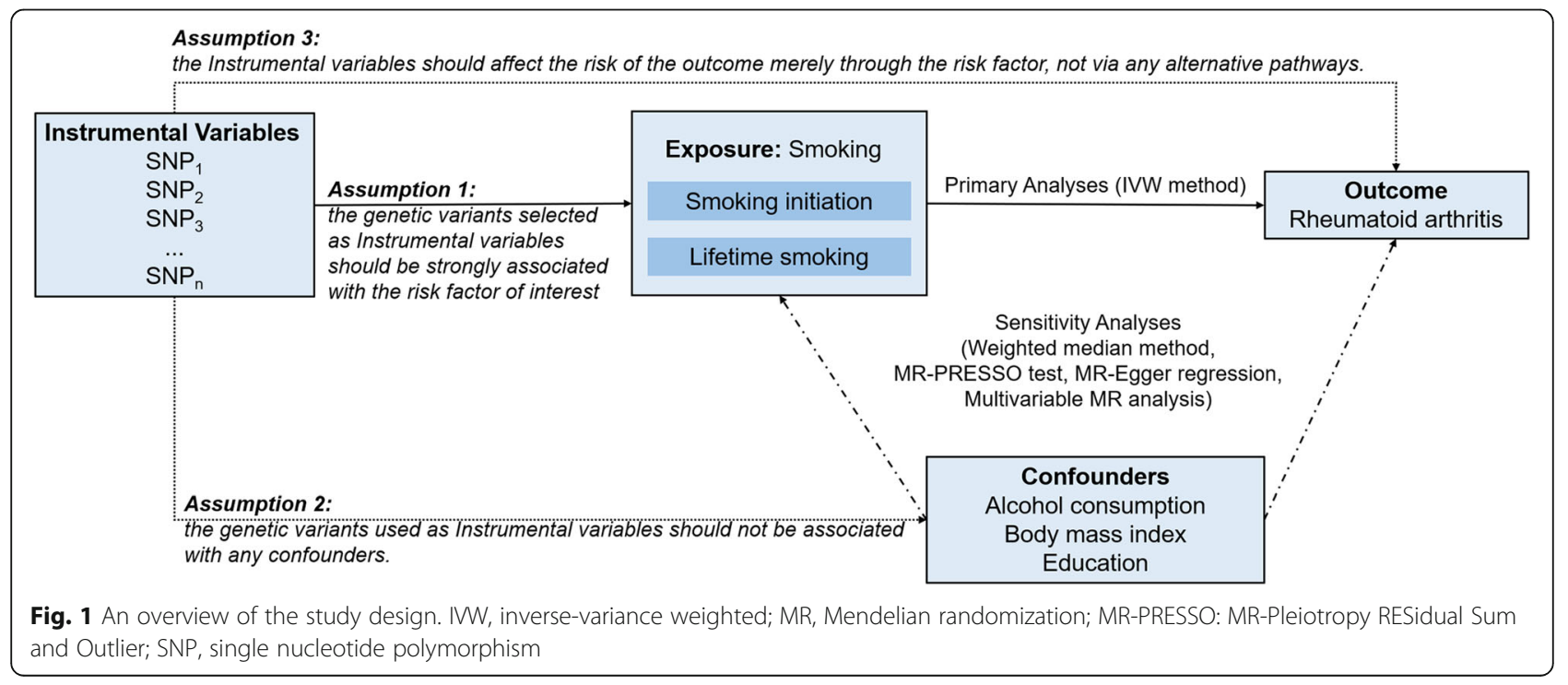




\section{Statistical analyses}

Statistical analyses were performed using the MendelianRandomization [10], and MR-PRESSO [11] packages in $\mathrm{R}$ software version 3.6.0 (https://www.r-project.org/), unless otherwise noted. All estimates were reported with two-tailed $P$ values. In the main analysis, we used the inverse-variance weighted (IVW) method based on a random-effects model and supplemented with the weighted-median method [12]. To test for potential pleiotropy, we performed the MR-Pleiotropy RESidual Sum and Outlier (MR-PRESSO) test [11] and MR-Egger regression [13]. Each of the SNPs used as IVs we scanned for its potential secondary phenotypes using the GWAS catalog (http://www.ebi.ac.uk/gwas, accessed on November 20,2019$)$, and sensitivity analyses were further performed excluding the SNPs associated with traits other than smoking. $F$-statistics were calculated to evaluate the strength of the IVs [14].

In addition, since smoking initiation is genetically correlated with education attainment $\left(r_{\mathrm{g}}=-0.40\right)$, alcohol consumption $\left(r_{\mathrm{g}}=0.34\right)$, and body mass index (BMI) $\left(r_{\mathrm{g}}=0.12\right)$ [8], we performed multivariable MR analysis including the SNPs associated with these exposures, along with SNPs for smoking initiation, to test for whether the association between smoking and RA remained statistically significant after adjustment for confounders [15].

\section{Results}

Supplementary Table 1 presents the 367 SNPs used as IVs for smoking initiation in our MR analysis. Compared with never smokers, genetically predicted smoking initiation was positively associated with an increased risk of RA (odds ratio $(\mathrm{OR})=1.32,95 \%$ confidence interval $\left.(C I)=1.15-1.52, P=9.17 \times 10^{-5}\right)$ using the IVW method (Fig. 2). Similar effect estimate was obtained using the weighted-median method $(\mathrm{OR}=1.44,95 \% \mathrm{CI}=1.21-$ $\left.1.72, P=5.83 \times 10^{-5}\right)$. MR-Egger regression analysis did not suggest evidence of horizontal pleiotropy ( $P$ intercept $=0.579$ ). Though three possible outlier SNPs were identified using the MR-PRESSO test, the effect estimate of the association between genetically predicted smoking initiation and risk of RA did not change markedly after outlier correction $(\mathrm{OR}=1.32,95 \% \mathrm{CI}=1.16-1.50, P=$ $\left.2.62 \times 10^{-5}\right)$.

We then scanned the SNPs used as IVs for their potential secondary phenotypes using the GWAS Catalog. A total of 60 SNPs associated with smoking initiation were found to be associated with other traits (Supplementary Table 2). Excluding these SNPs that are associated with traits other than smoking did not change the results essentially $(\mathrm{OR}=1.25,95 \%$ $\mathrm{CI}=1.07-1.47, P=0.004$ using the IVW method). In the multivariable MR analysis including the SNPs associated with alcohol consumption, education attainment, and BMI, the association between genetically predicted smoking initiation and risk of RA remained in the same pattern $(\mathrm{OR}=1.37,95 \% \mathrm{CI}=1.19-1.58$, $P=1.96 \times 10^{-5}$ ).

Similarly, we found that genetically predicted lifetime smoking was positively associated with an increased risk of RA $(\mathrm{OR}=1.55,95 \% \mathrm{CI}=1.13-2.14, P=0.007$ for the IVW method). The weighted-median method also produced a consistent effect estimate $(\mathrm{OR}=1.52,95 \% \mathrm{CI}=$ $1.02-2.26, P=0.039)$. After correction for outlier SNPs $(n=3)$, the association remained statistically significant (OR $=1.61,95 \% \mathrm{CI}=1.23-2.12, P=0.001$ in the MRPRESSO test). Additionally, there was no indication for directional pleiotropy ( $P$ intercept $=0.645$ in the MREgger regression).

\section{Discussion}

This is the first MR study to examine whether smoking is causally associated with risk of RA. Our study based on genetics provides evidence that smoking is causally associated with an increased risk of RA. Compared with

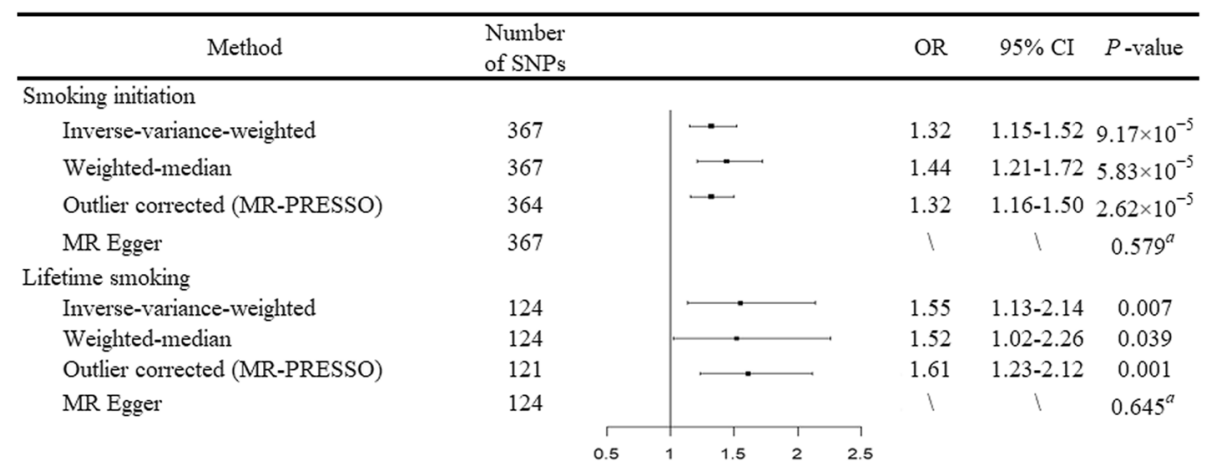

Fig. 2 Forest plot of Mendelian randomization analyses for the associations of smoking initiation and lifetime smoking with risk of rheumatoid arthritis. Cl, confidence interval; MR, Mendelian randomization; MR-PRESSO test, MR-Pleiotropy RESidual Sum and Outlier test; OR, odds ratio; SNP, single nucleotide polymorphism. ${ }^{\mathrm{a}} \mathrm{P}$ value of the intercept from MR-Egger regression analysis 
never smokers, genetic predisposition to smoking was associated with a $32 \%(95 \%$ CI $15 \%-52 \%)$ increased risk of developing RA. Furthermore, genetically predicted lifetime smoking was associated with a 55\% (95\% CI $13 \%-114 \%)$ increased risk of RA. These findings corroborate the results from the meta-analysis of observational epidemiological studies which showed that self-reported current smokers had a $40 \%$ increased risk of RA, compared to never smokers [3].

Although the underlying biological effect of smoking in the development of RA is still unclear [16], there are several plausible explanations. For example, studies have suggested that cigarette smoking can increase oxidative stress in the body through its content of oxidant gasses (e.g., free radicals) and other toxic substances (e.g., nicotine), which may increase the risk of RA through impaired antioxidant systems, platelet activation and inflammation [17]. In addition, chronic smoking can adversely affect the innate and adaptive immune responses and trigger various morphological, physiological, biochemical, and enzymatic changes that lead to impaired antibacterial defenses, cellular regulatory activity, and inflammatory responses, which may contribute to the development of RA [17]. Furthermore, it has been hypothesized that smoking could interact with $H L A-D R$ shared epitope genes and trigger HLA-DR-restricted immune reactions to autoantigens modified by citrullination [18]. Though these explanations are biologically plausible, further studies are warranted to elucidate the underlying mechanisms of smoking in the development of RA.

The major strength of this study is that the MR design allows us to investigate the causal nature of the association between smoking and RA. One limitation is that we were unable to stratify the analysis by sex and smoking status, and therefore could not assess gender discrepancies and potential nonlinear associations between smoking and risk of RA. Another limitation is that our analyses were restricted to participants of European ancestry; therefore, our results may not necessarily apply to populations of other ethnicities. However, this fact also reduces the potential bias caused by population stratification.

The validity of the MR approach relies on the following three key assumptions. First, the genetic variants selected as IVs should be strongly associated with the risk factor of interest; second, the genetic variants used as IVs should not be associated with any confounders; and third, the IVs should affect the risk of the outcome merely through the risk factor, not via any alternative pathways [19]. In the current study, we only used SNPs that are strongly associated with smoking initiation and lifetime smoking at genome-wide significance threshold. The F-statistics for smoking initiation and lifetime smoking was 79 and 13, respectively, which reduced the chance of weak instrument bias and the possible violation of the first assumption. As genotype is presumed to be randomly assorted at conception, covariates are anticipated to be randomly distributed with respect to genotype. However, it is possible that some SNPs used as IVs are associated with smoking as well as other traits if smoking is causally associated with these secondary phenotypes. It is also possible that some SNPs are associated with multiple pathways, including those not involving smoking. In the current study, we performed the MREgger regression and MR-PRESSO tests, which did not indicate the presence of directional pleiotropy. Sensitivity analyses using alternative MR methods including weighted-median and multivariable MR analyses also demonstrated a consistent association. In addition, we manually scanned each of the SNPs used as IVs for potential secondary phenotypes in the GWAS Catalog; MR analyses excluding these SNPs produced similar results.

\section{Conclusion}

In summary, using the MR approach, we have found that genetic predisposition to smoking is associated with risk of RA, suggesting that there is a causal relationship between smoking and the development of RA.

\section{Supplementary information}

Supplementary information accompanies this paper at https://doi.org/10. 1186/s13075-020-2134-1.

Additional file 1: Table S1. Effect estimates of the associations between the instrumental variables for smoking initiation and risk of rheumatoid arthritis. Abbreviations: Chr, Chromosome; RA, rheumatoid arthritis; SE, standard error; SNP, single nucleotide polymorphism. Table S2. The potential secondary phenotypes of the instrumental variables used for smoking initiation (from the GWAS catalog) a . Abbreviations: Chr, chromosome; EA, effect allele; SNP, single nucleotide polymorphism.

${ }^{a}$ Traits associated with the SNP according to previous genome-wide association studies.

\section{Abbreviations \\ Cl: Confidence interval; GWAS: Genome-wide association studies; IV: Instrumental variable; IWW: Inverse-variance weighted; MR: Mendelian randomization; MR-PRESSO: MR-Pleiotropy RESidual Sum and Outlier; OR: Odds ratio; RA: Rheumatoid arthritis; SNP: Single nucleotide polymorphism}

\section{Acknowledgements}

We thank all the participants and researchers of the studies included in the current analyses.

\section{Authors' contributions}

All authors interpreted the data, critically revised the manuscript, and approved the final version of the manuscript.

\section{Funding}

This work was supported by grants of the National Natural Science Foundation of China $(81973663,81873269$, and 81602917), the National Key R\&D Program of China (2018YFC1705500), and Talent Project of Zhejiang Association for Science and Technology (2018YCGC003). 


\section{Availability of data and materials}

Summary statistics for RA are available at http://plaza.umin.ac.jp/ yokada/ datasource/software.htm.

\section{Ethics approval and consent to participate}

Participating studies of the GWAS meta-analyses have received prior approval by relevant institutional review boards, and informed consent was obtained from each study participant. The current study was based on publicly available summary statistics data; therefore, no additional ethics approval was required.

\section{Consent for publication}

Not applicable.

\section{Competing interests}

The authors declare that they have no competing interests.

\section{Author details}

${ }^{1}$ School of Public Health, Zhejiang Chinese Medical University, Hangzhou 310053, Zhejiang, China. ${ }^{2}$ University of Minnesota Medical School,

Minneapolis, MN 55455, USA. ${ }^{3}$ Institute of Basic Research in Clinical Medicine, Zhejiang Chinese Medical University School of Basic Medical Sciences, Hangzhou 310053, Zhejiang, China. ${ }^{4}$ Department of Epidemiology \& Biostatistics, Zhejiang Chinese Medical University School of Basic Medical Sciences, Hangzhou 310053, Zhejiang, China.

Received: 10 December 2019 Accepted: 19 February 2020

Published online: 06 March 2020

\section{References}

1. Smolen JS, Aletaha D, McInnes IB. Rheumatoid arthritis. Lancet. 2016; 388(10055):2023-38.

2. Mclnnes IB, Schett $G$. Pathogenetic insights from the treatment of rheumatoid arthritis. Lancet. 2017;389(10086):2328-37.

3. Sugiyama D, Nishimura K, Tamaki K, Tsuji G, Nakazawa T, Morinobu A Kumagai S. Impact of smoking as a risk factor for developing rheumatoid arthritis: a meta-analysis of observational studies. Ann Rheum Dis. 2010; 69(1):70-81.

4. Di Giuseppe D, Discacciati A, Orsini N, Wolk A. Cigarette smoking and risk of rheumatoid arthritis: a dose-response meta-analysis. Arthritis Res Ther. 2014; 16(2):R61.

5. Emdin CA, Khera AV, Kathiresan S. Mendelian randomization. JAMA. 2017; 318(19):1925-6.

6. Okada Y, Wu D, Trynka G, Raj T, Terao C, Ikari K, Kochi Y, Ohmura K, Suzuki A, Yoshida $\mathrm{S}$, et al. Genetics of rheumatoid arthritis contributes to biology and drug discovery. Nature. 2014;506(7488):376-81.

7. Arnett FC, Edworthy SM, Bloch DA, McShane DJ, Fries JF, Cooper NS, Healey LA, Kaplan SR, Liang MH, Luthra HS, et al. The American Rheumatism Association 1987 revised criteria for the classification of rheumatoid arthritis. Arthritis Rheum. 1988;31(3):315-24.

8. Liu M, Jiang Y, Wedow R, Li Y, Brazel DM, Chen F, Datta G, Davila-Velderrain J, McGuire D, Tian C, et al. Association studies of up to 1.2 million individuals yield new insights into the genetic etiology of tobacco and alcohol use. Nat Genet. 2019;51(2):237-44.

9. Wootton RE, Richmond RC, Stuijfzand BG, Lawn RB, Sallis HM, Taylor GMJ, Hemani G, Jones HJ, Zammit S, Smith GD, et al. Causal effects of lifetime smoking on risk for depression and schizophrenia: evidence from a Mendelian randomisation study. Psychol Med. 2019;1-9. https://doi.org/10. 1017/S0033291719002678. https://www.cambridge.org/core/product/ identifier/S0033291719002678/type/journal_article.

10. Yavorska OO, Burgess S. MendelianRandomization: an R package for performing Mendelian randomization analyses using summarized data. Int J Epidemiol. 2017:46(6):1734-9.

11. Verbanck M, Chen CY, Neale B, Do R. Detection of widespread horizontal pleiotropy in causal relationships inferred from Mendelian randomization between complex traits and diseases. Nat Genet. 2018;50(5):693-8.

12. Bowden J, Davey Smith G, Haycock PC, Burgess S. Consistent estimation in Mendelian randomization with some invalid instruments using a weighted median estimator. Genet Epidemiol. 2016;40(4):304-14.
13. Bowden J, Davey Smith G, Burgess S. Mendelian randomization with invalid instruments: effect estimation and bias detection through Egger regression. Int J Epidemiol. 2015;44(2):512-25.

14. Burgess S, Thompson SG, Collaboration CCG. Avoiding bias from weak instruments in Mendelian randomization studies. Int J Epidemiol. 2011;40(3): 755-64.

15. Burgess S, Thompson SG. Multivariable Mendelian randomization: the use of pleiotropic genetic variants to estimate causal effects. Am J Epidemiol. 2015 181(4):251-60.

16. Di Giuseppe D, Orsini N, Alfredsson L, Askling J, Wolk A. Cigarette smoking and smoking cessation in relation to risk of rheumatoid arthritis in women. Arthritis Res Ther. 2013;15(2):R56.

17. Chang K, Yang SM, Kim SH, Han KH, Park SJ, Shin Jl. Smoking and rheumatoid arthritis. Int J Mol Sci. 2014;15(12):22279-95.

18. Klareskog L, Padyukov L, Alfredsson L. Smoking as a trigger for inflammatory rheumatic diseases. Curr Opin Rheumatol. 2007;19(1):49-54.

19. Davies NM, Holmes MV, Davey Smith G. Reading Mendelian randomisation studies: a guide, glossary, and checklist for clinicians. BMJ. 2018;362:k601.

\section{Publisher's Note}

Springer Nature remains neutral with regard to jurisdictional claims in published maps and institutional affiliations.
Ready to submit your research? Choose BMC and benefit from:

- fast, convenient online submission

- thorough peer review by experienced researchers in your field

- rapid publication on acceptance

- support for research data, including large and complex data types

- gold Open Access which fosters wider collaboration and increased citations

- maximum visibility for your research: over $100 \mathrm{M}$ website views per year

At BMC, research is always in progress.

Learn more biomedcentral.com/submissions 